\title{
Literaturbericht
}

\author{
Hubert Zimmermann
}

\section{Die Politikwissenschaft, der Euro und die Staatsschuldenkrise*}

Seit mehr als zwei Jahren ist die Schuldenkrise in der Eurozone das beherrschende Thema der Weltwirtschaft und insbesondere der europäischen Politik. Wie können die Entstehung, der Verlauf und die Konsequenzen dieser schwersten Erschütterung des europäischen Integrationsprozesses seit der Begründung der supranationalen Zusammenarbeit in Europa erklärt werden? Was bedeutet die radikale Infragestellung der Bonität von Staatsanleihen westlicher Länder für eine internationale politische Ökonomie, die auf staatlichen Schuldverschreibungen als ultimativer Garantie ökonomischer Sicherheit beruht? Noch ist es zu früh für eine wirklich profunde, quellengesättigte und theoretisch sowie methodisch abgesicherte Analyse, zumal die Krise noch im Gange ist. Auch hat sich noch keine dominante Erzählung etabliert, weder in der öffentlichen Debatte noch in der Wissenschaft. Think-Tanks und die Leitartikler der führenden Qualitätspresse in Europa produzieren täglich neue Einschätzungen. Auch die Politikwissenschaft ist von den Ausmaßen der Krise überrascht worden und weit entfernt von einer abschließenden Bewertung. Dies mag daran liegen, dass die Krise unterschwellige Kontroversen und fundamentale Gegensätze im Kern der politischen und ökonomischen Prozesse im Europa der letzten Jahrzehnte in ein scharfes Licht gerückt hat und dazu führte, dass viele scheinbar unumstößliche Gewissheiten plötzlich in Frage stehen (Zimmermann/Dür 2012). Ähnlich wie die Disziplin der Internationalen Beziehungen sich nach dem Ende des Kalten Krieges neu aufstellen musste, so steht auch die politikwissenschaftliche Europaforschung und die verwandten Disziplinen, die sich mit der EU beschäftigen, jetzt vor der Herausforderung, dass viele der bisherigen wissenschaftlichen Steckenpferde plötzlich nicht mehr sehr bedeutsam erscheinen. Andere, bis dahin wenig bearbeitete Themen erhalten hingegen eine enorme Relevanz. Die Kontroversen

* Für ausgezeichnete Kommentare danke ich Martin Höpner vom Max-Planck-Institut für Gesellschaftsforschung (MPIfG) Köln und Andreas Nölke (Goethe-Universität Frankfurt/Main). 
in der Europaforschung werden, wie diejenigen in der aktuellen Europapolitik, schärfer und politischer werden (müssen).

Der vorliegende Beitrag versucht eine erste vorläufige Bestandsaufnahme der Literatur zur Eurokrise, wobei ein Großteil der Debatte bis jetzt noch nicht in der genuin wissenschaftlichen Literatur Eingang gefunden hat, sondern sich in der Qualitätspresse, Publikumszeitschriften und Think-Tank-Publikationen abspielt. Deshalb werden hier vor allem Grundfragen skizziert, mit denen sich politikwissenschaftliche Forschungen erst noch in systematischer Art werden auseinandersetzen müssen und die traditionelle, oft schon angestaubte, Forschungsagenden entweder wieder aufgreifen oder neu aufreißen.

Eine der wichtigsten Konsequenzen der Krise ist wohl das Kippen der pauschal positiven Bewertung des europäischen Integrationsprozesses, der im regionalen Bereich die Lösung globaler Kooperationsprobleme vorwegzunehmen schien, die der Ansatz der Global Governance im weltweiten Maßstab suchte, dabei aber nur in bestimmten Bereichen wirklich fündig wurde. Gerade in der Politikwissenschaft galt die EU immer als das Musterbeispiel für das „Regieren in entgrenzten Räumen“ und die erhoffte Überwindung des nationalstaatlichen Paradigmas. Deshalb hielt sich der vielzitierte permissive Konsensus (Lindberg/Scheingold 1970), der dem Integrationsprozess so lange ein scheinbar festes Fundament bot, dort auch viel länger als außerhalb des akademischen Elfenbeinturms. Dies gilt insbesondere für die politikwissenschaftliche Europaforschung. Die EU war zu einer Selbstverständlichkeit geworden, der zwar immer weniger die emotionale Wärme der früheren Europabegeisterung entgegengebracht wurde, mit der man sich aber insgesamt gut eingerichtet hatte. Das Hauptaugenmerk galt den Funktionsmerkmalen dieser ungewöhnlichen Polity, nicht aber ihrer raison d'être sowohl im politischen als auch ökonomischen Bereich. Zwar wurde die Debatte um das sogenannte demokratische Defizit schon seit dem Maastrichter Vertrag 1992 intensiv geführt. Aber hierbei handelte es sich doch - wie beim Großteil der sich immer weiter ausdifferenzierenden und sich mit Lehrstühlen und Forschungsverbünden institutionalisierenden Europaforschung - um die Frage, wie die EU zu verbessern sei, nicht aber darum, ob die EU und ihre spektakulärste Errungenschaft, die Währungsunion, an sich ein öffentliches Gut sei. Fundamentale Kritik an der EU war immer mit dem Stigma besetzt, dass sie öffentlich vor allem von Exponenten der Ränder des politischen Spektrums geäußert wurde. So umgab eine Ablehnung der gemeinsamen europäischen Währung immer der Ruch des Reaktionären, ob es sich nun um eine Verteidigung der reinen Lehre der deutschen Geldpolitik handelte, wie bei dem bekannten 
Aufruf der deutschen Volkswirtschaftsprofessoren, ${ }^{1}$ oder um die Klagen konservativer Politiker vor dem Bundesverfassungsgericht. Ein ähnliches Stigma behaftete die linke Extremposition, in der der Euro in seiner letztlichen Form als neoliberales Projekt einer transnationalen Finanzelite kritisiert wurde (van Apeldoorn/Drahokoupil/Horn 2009), eine Sichtweise, die durch die Sparpolitik in vielen Euroländern einen enormen Auftrieb erhalten hat und sich in zunehmenden Wahlerfolgen einer europaskeptischen Linken außerhalb Deutschlands äußert. Seit der Einführung der gemeinsamen Währung und insbesondere in jüngster Zeit nahm auch die Unterstützung der europäischen Bevölkerung für die europäische Integration rapide ab, wie neue Meinungsumfragen belegen (Pew Research Center 2012). Europa wird inzwischen mit dem Euro gleichgesetzt, und die Konnotation ist zunehmend negativ.

Die politikwissenschaftliche Europaforschung hat die politischen Kernfragen des Projekts der Währungsunion viel zu lange ignoriert, wie Jürgen Kaube jüngst in der FAZ zurecht festgestellt hat: „Das Licht der Europa-Forschung strahlt derzeit auch darum so schwach, weil sie jetzt wieder zu dem zurückkehren muss, was Europa angeblich nicht nur war, was sich aber als Bedingung für alles Weitergehende darstellt: seiner Politischen Ökonomie.“ (Kaube 2012) In der Tat befassten sich die intensivsten politikwissenschaftlichen Debatten nicht mit Fragen der politischen Ökonomie der EU, wie es bei der Natur des europäischen Zusammenschlusses zu erwarten gewesen wäre. Selbst auf der 12. Jahrestagung der European Union Studies Association (EUSA) im März 2011, mitten in der Staatsschuldenkrise, waren Panels, die sich mit den wirtschaftlichen Grundfragen des europäischen Projekts beschäftigten, eindeutig in der Minderzahl gegenüber jenen, die policy-making, governance, identity, public opinion etc. als Fokus angaben. Dies gilt auch für Beiträge in den führenden EU-Zeitschriften, wie dem Journal of Common Market Studies, Journal of European Integration oder Journal of European Public Policy. Seit dem Governance Turn haben die genannten Themen die zentrale Frage nach den Entstehungsbedingungen der Union abgelöst, die noch im Fokus der Neofunktionalismus-Intergovernmentalismus-Debatte stand. Die Forschung über Phänomena wie die zunehmenden grenzüberschreitenden Kapitalflüsse und Investitionen in der EU, der Konvergenzdruck auf europäische Volkswirtschaften, die Finanzialisierung der europäischen Wirtschaft und die politischen Ursachen solcher und ähnlicher Prozesse fristete dagegen weiter ein Nischendasein. Dies führte dazu, dass die weithin bekannten und konstatierten Probleme der Währungsunion in ihrer Virulenz unterschätzt wurden.

1 Siehe den Aufruf von 155 Wirtschaftswissenschaftlern „Der Euro kommt zu früh“ in der Frankfurter Allgemeinen Zeitung vom 9. Februar 1998. 
Allerdings sind auch diejenigen Politikwissenschaftler, die sich spezifisch mit dem Euro befassten (wie der Autor dieser Zeilen), vom Ausbrechen der Krise und ihren Ausprägungen überrascht wurden. Oder, präziser ausgedrückt: Die allseits konstatierte Lückenhaftigkeit des Europrojekts zog zwar eine breite Palette von Reformvorschlägen nach sich, aber es wurde allgemein erwartet, dass deren Umsetzung inkrementell erfolgen würde (z. B. Eichengreen 2010) und nicht in einer Art und Weise, die die meisten Mitgliedstaaten eines großen Teils ihrer Handlungsautonomie beraubt und den Sinn des europäischen Projekts insgesamt in Frage stellen könnte. Die Literatur zum Euro ${ }^{2}$ hatte sich zunächst mit den Bedingungen des Zustandekommens der gemeinsamen Währung auseinandergesetzt (Dyson/Featherstone 1999; McNamara 1998; Moravcsik 1998; Verdun 2000) und nur gelegentlich mit der Politikgestaltung in diesem Politikfeld (Dyson 2000). Erst in den letzten Jahren wurde die Funktionsweise der wichtigsten Institutionen der Eurozone politikwissenschaftlich untersucht. ${ }^{3}$ Die Aushöhlung des Stabilitäts- und Wachstumspaktes war ein erster Testfall für die Funktionsfähigkeit dieser unfertigen, wesentlich auf freiwilligen Selbstverpflichtungen beruhenden Währungsunion (Heipertz/ Verdun 2010). Sie wurde zumeist als bedauerlicher und ernster Verstoß gesehen, nicht aber als Beleg, dass die Idee der gemeinsamen Währung vielleicht doch verfrüht war, wenn sogar der Kern der Eurozone so schnell die Regeln brach. Die Bestandsaufnahmen zum zehnjährigen Bestehen des Euro unterschieden sich dann in der Grundtendenz nicht sehr vom Ton der Berichts der Europäischen Kommission zum Jubiläum, in dem der Euro als resounding success bezeichnet wurde (European Commission 2008: 3). In einem Sonderheft des Journal of European Public Policy ließen 2009 einige der ausgewiesendsten ExpertInnen des Euro die politikwissenschaftliche Forschung zum Thema Revue passieren und wagten einen Ausblick in die Zukunft. Enderlein und Verdun (2009) konstatierten, dass die Eurozone trotz ihrer asymmetrischen Struktur, in der eine Währungsunion durch keine politische Union ergänzt wurde, ökonomisch funktioniert habe und auch keine gravierenden Legitimationsdefizite aufweisen würde. Ein Spillover von der Währungsunion in Richtung auf eine Art Wirtschaftsregierung (gouvernement économique) sei nicht festzustellen. Die Krise hat diese Diagnose gründlich über den Haufen geworfen. Woran liegt dies?

Ein zentraler Grund ist sicher, dass die grundlegende Logik der Eurozone nicht ernst genug genommen wurde und die im relativ ruhigen wirtschaftlichen Umfeld des ersten Eurojahrzehnts eingebetteten Fehlentwicklungen unterschätzt wurden.

2 Sadeh und Verdun (2009) bieten einen Überblick bis zur Finanzkrise.

3 Zur Eurogruppe vergleiche Puetter 2006; zur EZB vergleiche Howarth/Loedel 2000. 
Ian Cooper (2011) hat die Eurokrise vor einigen Monaten als „Rache des Neofunktionalismus" bezeichnet. Er deutete damit an, dass die Einführung des Euro eine massive, teil verborgene Spillover-Dynamik in Gang gesetzt hatte, die sich jetzt in der Krise Bahn bricht. Nur wenige Autoren haben sich aber im letzten Jahrzehnt mit der Weiterentwicklung der wenig angesagten neofunktionalistischen Theorie und deren Anwendung auf die Währungsunion beschäftigt. Dies wird sich ändern. Des Weiteren hätte auch eine vertiefte Auseinandersetzung mit den Ergebnissen der vergleichenden Kapitalismusforschung sowie der kritischen Literatur zur Finanzialisierung ${ }^{4}$ vielleicht den Blick für Dynamiken geschärft, die dann plötzlich in so unerwartetem Ausmaß ausbrachen. Schon früh hat Philip Schmitter auf die Bedeutung der Unterschiede in nationalen Produktionssystemen für die europäische Konstruktion hingewiesen und die Frage gestellt, wie sich unter diesen Umständen eine Konvergenz nationaler Systeme vollziehen könnte (Schmitter 1997). David Bearce (2009) hat vor Beginn der Krise gezeigt, dass diese Konvergenz wider Erwarten keine Folge der Einführung der gemeinsamen Währung war. Für PolitikwissenschaftlerInnen, die sich aus der Perspektive der vergleichenden politischen Ökonomie $^{5}$ mit der europäischen Integration beschäftigten, war das keine Überraschung, da sie schon immer die auffallende Pfadabhängigkeit unterschiedlicher Kapitalismusmodelle untersuchten. Die Literatur zur Finanzialisierung wiederum betont, wie die zunehmende Bedeutung von auf finanziellen Prozessen basierendem künstlichem Wachstum gegenüber realwirtschaftlichem Wachstum zu einer unerwarteten Interdependenz der europäischen Volkswirtschaften und zur Aushebelung legitimer Steuerungsmechanismen führt (Nölke/Heires 2011). Eine weiterhin sehr heterogene Währungsunion verlor so die Kapazität, auf eventuell unkontrollierte Anpassungsprozesse, die von der Währungsunion in Gang gesetzt wurden, steuernd einzuwirken. Seit 2007 wurde dies immer offensichtlicher. Schien die erste Phase der Finanzkrise noch im Großen und Ganzen eine mit traditionell intergouvernementalen Mitteln zu bewältigende Herausforderung, so hat der kumulative Herdentrieb der Finanzmärkte spätestens mit dem Scheitern des ersten Rettungspakets für Griechenland im Frühjahr 2010 die Handlungsfähigkeit der EU radikal in Frage gestellt.

Eine der ersten soliden Darstellungen dieser Entwicklungen hat der englische Journalist Matthew Lynn (2011) vorgelegt. Die flott geschriebene und oft ins Stereotype abgleitende Darstellung bietet eine gleichwohl gut informierte Schilderung der Krise bis Mitte 2010. Lynn gibt dabei die konventionelle Darstellung der Kri-

$4 \mathrm{Zu}$ Ursachen und Auswirkungen der Finanzialisierung forscht seit 2010 ein von der DFG gefördertes Netzwerk. Vergleiche http://www.finanzialisierung.net/startseite (Stand: 7.5.2012).

5 Einen Überblick liefert Höpner 2009. 
senursachen wieder. Danach liegt eine erste Ursache in den historisch bedingten Dysfunktionalitäten des griechischen Staates, die dazu führten, dass die Chancen, die der Beitritt zur EU und zum Euro bot, leichtfertig verspielt wurden. Dazu gehören innenpolitische Reformblockaden, die durch eine politische Kultur perpetuiert wurden, in der Klientelismus und Korruption weit verbreitet sind (Featherstone 2011). Schon Reinhart und Rogoff haben in ihrem ungemein einflussreichen und vielzitierten historischen Abriss über den Zusammenhang von Finanz- und Staatsschuldenkrisen darauf hingewiesen, dass Griechenlands Geschichte von einer Abfolge von Staatsbankrotten gekennzeichnet war (Reinhart/Rogoff 2009: 98). Die bisher nur wenig untersuchte Ausweitung der Eurozone von ursprünglich 11 auf mittlerweile 17 Mitglieder vollzog sich mittels eines Automatismus, der nur wenig Raum ließ für eine vertiefte Erörterung der Frage, ob Neumitglieder - wie Griechenland - tatsächlich die ökonomischen Grundlagen, vor allem aber die institutionelle Steuerungsfähigkeit aufwiesen, um die notwendigen Anpassungsleistungen zu ergreifen (Zimmermann 2012 b). Durch den Wegfall der Abwertungsoption nach dem Euro-Beitritt hatte Griechenland jedenfalls das zentrale Instrument verloren, welches einem blockierten politischen System zur Wiedererlangung der Wettbewerbsfähigkeit zur Verfügung stand. Die genauen Mechanismen, die dazu geführt haben, dass die Aufnahme Griechenlands in die EU, die aufgrund der (vielleicht nur vordergründigen) Stabilisierung der Demokratie meist als Erfolgsgeschichte geschrieben wurde, nicht in einer gesellschaftlichen und politischen Modernisierung resultierte und die Frage, weshalb die günstigen wirtschaftlichen Rahmenbedingungen, die der Euro bereitstellte, nicht genutzt wurden, müssen sicher noch untersucht werden. Dies ist eine große Herausforderung für die Europäisierungsforschung (Graziano/Vink 2006; Kohler-Koch/Eising 1999).

Welches sind die Bedingungen der langfristigen Institutionalisierung jener oft vordergründigen Reformen, die zum erfolgreichen Euro-Beitritt so vieler Wackelkandidaten beigetragen haben, und wie laufen die notwendigen Einstellungsänderungen ab bzw. durch was werden sie verhindert? Wie europäisieren sich öffentliche Verwaltungen und pfadabhängige Strukturen nationaler politischer Ökonomien? Offensichtlich handelt es sich dabei um Prozesse, die erheblich langsamer vonstattengehen, als es die Hoffnung einer schnellen Konvergenz von Seiten vieler EuroOptimisten vermuten ließ. Seit den ersten Plänen für eine europäische Währungsunion gehört ja die mangelnde Flexibilität des Währungsraums beim Ausgleich asymmetrischer Schocks zu den Kernargumenten vieler Euroskeptiker, die davor warnten, dass die Eurozone eben kein optimaler Währungsraum sei (de Grauwe 2000). Diese Stimmen fühlen sich jetzt bestätigt. Dazu gehört auch die These, dass es gerade die Konsequenzen des Euro-Beitritts waren, vor allem die niedrigen Zin- 
sen auf Staatsanleihen, die den Reformdruck von den griechischen Regierungen nahmen. Letztlich waren es aber auch die Konstruktionsfehler des Euro selbst, die hauptverantwortlich für die Krise waren, wie Fritz Scharpf (2011) in einer der tiefgründigsten Analysen der Krise feststellt. Demnach verursachte der Euro die destabilisierenden makroökonomischen Ungleichgewichte und die enorme Verwundbarkeit vieler europäischer Staaten gegenüber den Folgen finanzieller Krisen. Verhängnisvoll sei vor allem, dass die Eurozone demokratisch gewählten Regierungen einige der wichtigsten Instrumente zum Management wirtschaftlicher Verwerfungen entzogen hat und es Staaten damit erheblich erschwert, den Krisenfolgen entgegenzusteuern. Scharpf befürchtet als Folge einen nicht kontrollierbaren Verlust der Legitimität europäischen Regierens. Im kleineren Umfang sei dies in Deutschland schon geschehen. Hohe Realzinsen hatten die deutsche Wettbewerbsfähigkeit in der Eurozone zunächst untergraben (siehe auch Busch 2010). Durch mit den Sozialpartnern koordinierte Lohnzurückhaltung konnte diese aber zurückgewonnen werden. Der Preis war allerdings ein erheblicher Legitimationsverlust des politischen Systems (Scharpf 2011: 176) sowie eine Verschlechterung der Wettbewerbsfähigkeit der anderen EU-Mitgliedstaaten in Relation zu Deutschland.

Das deutsche Beharren darauf, die gewonnenen Wettbewerbsvorteile zu wahren und die Anpassungsleistungen auf die Defizitländer abzuwälzen, hat dazu geführt, dass vielfach auch Deutschland eine erhebliche Mitverantwortung an der Eskalation der Krise zugeschrieben wurde. Eric Jones (2010) hat schon früh die zögerliche Haltung der Merkel-Regierung kritisiert und dies auf innenpolitische Faktoren zurückgeführt (vgl. auch Zimmermann 2012 a). Blyth und Matthijs (2011) sehen die historisch bedingte Zurückhaltung der deutschen Außenpolitik als Ursache für die Zuspitzung der Krise. Deutschland müsse, analog zur Rolle der USA im BrettonWoods-System, als ,,aufgeklärter Hegemon“ die öffentlichen Güter produzieren, die notwendig sind, um eine Konstruktion wie die Eurozone abzusichern. Dazu gehören die Bereitstellung eines Marktes für überschüssige Güter, antizyklische Langzeitdarlehen, stabile Wechselkurse, eine führende Rolle bei der makroökonomischen Koordination sowie die Akzeptanz der Rolle als Lender of Last Resort. Dass Deutschland diese Rolle nicht einnehme, liegt nach Ansicht von Brigitte Young und Willi Semmler (2011) aber nicht an einer Kultur der Zurückhaltung, sondern vielmehr an der stärkeren Betonung nationaler Interessen durch Deutschland und einer schrittweisen Abkehr vom reflexiven Multilateralismus. Die Krise hat, wie diese Stellungnahmen zeigen, auch die traditionelle Debatte über die deutsche Außenpolitik nach dem Kalten Krieg neu befruchtet, dokumentiert auch durch eine kürzlich erschienene Ausgabe von Aus Politik und Zeitgeschichte (Bundeszentrale für politische Bildung 2012) zum Thema. 
Deutschlands Rolle in der Krisenbewältigung wird ein zentrales Thema der Europaforschung der nächsten Jahre sein. Auch die zahlreichen Regierungswechsel in Deutschlands Partnerländern werden ja auf die scharf kritisierte deutsche Austeritätspolitik zurückgeführt. Durch die zunehmenden Proteste gegen diese Politik kann sich auch die kritische Europaforschung, die bisher ein wenig beachtetes Randdasein führte, bestätigt fühlen. Danach entsprach die Eurozone einem hegemonialen Projekt transnationaler Eliten, welches die neoliberale Ausrichtung der Währungsunion im Sinne ihrer Interessen bestimmte (Bieling 2011). Die Folge war ein massiver Verlust an demokratischer Selbstbestimmung an die Märkte: ,the Maastricht criteria and successive financial pacts have firmly subordinated macro-economic policy to short-term global financial markets"(Cafruny/Ryner 2009: 233). Die Krise hätte nun aber Gegenkräfte mobilisiert, die, gegebenenfalls sogar in Form eines „europäischen Frühlings“, alternativen Ordnungsvorstellungen auf europäischer Ebene zum Durchbruch verhelfen könnte (Staatsprojekt Europa 2012). Allerdings muß konstatiert werden, dass die europaweite Vernetzung der verschiedenen Protestbewegungen nicht erfolgt und deren Interessen durchaus heterogen sind. Auch fehlt auf europäischer Ebene ein Ansprechpartner, da das Europäische Parlament in der Krise bisher kaum in Erscheinung getreten ist. Allerdings weist die kritische Europaforschung zu Recht auf die zunehmende Erosion des „,neoliberalen“ Konsenses hin. Das verweist auf die Notwendigkeit, die Ideen, auf denen die Konstruktion der gemeinsamen Währung beruhte, sowie deren Wandel präziser als bisher zu bestimmen (Blyth/Abdelal/Parsons 2010).

Während die griechische Situation die europäische Politik vor das Dilemma stellte, fundamentale Grundsätze, auf die der Euro gegründet war, über Bord zu werfen, bedeutete die Ausweitung der Krise auf andere Euro-Staaten, insbesondere die Schwergewichte Spanien und Italien, eine existenzielle Bedrohung für das Überleben des Euro. Dabei handelte es sich bei Ländern wie Spanien und Irland weniger um ein Problem der Staatsschulden als um eine Folge des Platzens der Spekulationsblasen in der globalen Finanzkrise. Schien die EU zunächst noch durchaus in der Lage, die Folgen des Lehman-Zusammenbruchs besser zu bewältigen als die USA (Gottwald 2012; Zimmermann 2009; dagegen aber Mügge 2012), so zeigte die Staatsschuldenkrise in aller Deutlichkeit die Lücken in der Währungsunion auf. Schon in ihrer Bestandsaufnahme von 2008 hatten Dyson u. a. zum Beispiel konstatiert, dass der Euro nicht zu einer Zentralisierung und Konvergenz der Finanzmarktaufsicht geführt habe (Dyson 2008: 21). Laut Eichengreen (2012) war das Fehlen einer effektiven, supranationalen Finanzmarktaufsicht ein zentraler Faktor, weshalb die Krise die Verantwortlichen in Europa so unvorbereitet traf. Die Finanzmarktaufsicht ist nur ein Beispiel, welches die Folgen des Fehlens der schon 
bei den ersten Plänen zur Wirtschafts- und Währungsunion so heftig geforderten, aber immer gescheiterten ,wirtschaftlichen“ Komponente der WWU (Mechanismen zum Lastausgleich, Fiskalunion etc.) zeigt. Stattdessen hoffte man über letztlich freiwillige Selbstverpflichtungen wie den Stabilitäts- und Wachstumspakt, eine gemeinsame Wirtschaftspolitik zu erreichen. Doch die vielzitierten neuen Formen des Regierens in Europa (Heritier/Rhodes 2011), die gerade hier hätten von Bedeutung werden können, scheinen in ihrer Wirksamkeit für die Eurozone weit überschätzt (Hodson 2011: 135 f.). Der Gedanke, die ordnungspolitische Grundphilosophie der Eurozone in ein festes, sanktionsfähiges Regelwerk zu überführen, bot sich demzufolge an und bildet zur Zeit den Tenor der deutschen und europäischen Antwort auf die Krise.

So haben die Maßnahmen, auf die sich die europäischen Regierungen geeinigt haben, zur rapiden Vergemeinschaftung der Wirtschaftspolitik geführt, die sicher nicht mehr als inkrementell (so Salines/Glöckler/Truchlewski 2012) bezeichnet werden. Die Rettungspakete für Griechenland mit den begleitenden Eingriffsrechten in nationale Souveränität, die vorläufigen Rettungsschirme EFSM und EFSF, der dauerhafte Mechanismus (ESM), die europaweite Verabschiedung von Schuldenbremsen, der von der Kommission vorgeschlagene „Six-Pack“, das „europäische Semester" usw. haben innerhalb kürzester Zeit zu einer Institutionalisierung supranationaler wirtschaftlicher Governance in der Eurozone geführt, deren letztendliches Ausmaß und Auswirkungen noch kaum abzusehen sind (Scheller 2012; Schwarzer 2012). Dabei handelt es sich um einen nachgeholten massiven SpilloverProzess, der die von vielen Wirtschaftswissenschaftlern als unfertig kritisierte Eurozone mit funktional notwendigen Instrumenten ausstattet (Beckert/Streek 2012: 14). Dazu gehört zum Beispiel eine europäische Finanzmarktaufsicht, die in der Lage ist, grenzüberschreitende Risiken zu erkennen und entsprechende Warnsignale zu senden. Allerdings werden auch diese neuen Institutionen weiterhin mit dem Grundproblem der Eurozone zu kämpfen haben: die nicht vorhandene Konvergenz der politischen Ökonomien und der Konjunkturzyklen (Höpner/Schäfer 2010). Eine zentrale Feststellung, die sich aus der vergleichenden Kapitalismusforschung ableiten läßt, ist, dass die Erweiterung der Eurozone und die damit einhergehende zunehmende Heterogenität der Volkswirtschaften entweder auf Kosten einer notwendigerweise raschen Vertiefung der Integration über eine Fiskalunion oder ähnliches oder aber zu Lasten der notwendigen demokratischen Legitimität geht (Höpner/Schäfer/Zimmermann 2012). Nur eine von oben durchgesetzte rasche Konvergenz scheint zum jetzigen Zeitpunkt in der Lage, die gemeinsame Währung zu stabilisieren. Allerdings scheint es ein Irrweg zu sein, diese Konvergenz durch gemeinschaftliche Vorgaben für alle Mitglieder zu erreichen, da die höchst unter- 
schiedlichen Bedingungen in den jeweiligen Staaten doch eher partikulare Maßnahmen erfordern. ${ }^{6}$

Aus der forcierten Supranationalisierung der Wirtschaftspolitik ergibt sich zudem ein gravierendes Problem im Hinblick auf die demokratische Legitimität dieser Maßnahmen. Diese Problematik, von Giandomenico Majone jüngst als „Integration by Stealth“ bezeichnet (2005), kennzeichnete Integrationsschritte, die auf Spillover und dem agency slack (unabhängige Aktionen des Agenten in einer PrincipalAgent-Beziehung) supranationaler Akteure beruhen, schon immer. Es scheint jedoch, als ob unter dem Kontingenzdruck der Krise nunmehr eine Schwelle überschritten worden ist, an der die weitere Abgabe von Kompetenzen an die supranationale Ebene dazu führt, dass die bisher nur in Großbritannien wirklich wahlentscheidende europäische Frage zu einer eben solchen auch in allen anderen Mitgliedstaaten wird, und zwar, weil Europa nun für alle Bürgerinnen und Bürger sichtbar in die essentiellen Grundlagen der europäischen Demokratien eingreift. Die teils gewaltsamen Proteste gegen die von außen oktroyierte Austeritätspolitik der Schuldnerstaaten, die zunehmenden Erfolge euroskeptischer Parteien, die Installation ,technokratischer" Regierungen mit sehr dünner Legitimität, die Warnungen des Bundesverfassungsgerichts vor der Aushöhlung der parlamentarischen Kontrolle (Voßkuhle 2012), das vernehmbare Aufbegehren im Bundestag gegen immer neue Ermächtigungen sowie die Flut EU-feindlicher Publikationen deuten hierauf hin. Der sich andeutende verschärfte Trade-off zwischen der Beschneidung der nationalen Souveränität und der Effektivität der nationalen Demokratie wird zu den wichtigsten Fragestellungen gehören, denen sich die Europaforschung in den nächsten Jahren zu stellen hat (Decker 2011). Die dabei entstehenden Konfliktlinien werden nicht nur zwischen den Lagern, sondern auch innerhalb dieser geführt werden (Nölke 2012).

Welche Verschiebungen zwischen intergouvernementaler und supranationaler Methode werden sich ergeben? Handelt es sich um eine EU im „Notstandsmodus“ (Rödl 2012), in dem die supranationalen Institutionen (Kommission, EZB, EuGH) endgültig die Demokratie aushöhlen? Oder ist die Ausstattung der EU mit weitreichenden Kompetenzen im Hinblick auf nationale Haushalte und Wirtschaftspolitik unausweichlich, um die bisherigen Errungenschaften der EU zu bewahren, wie eine „Große Koalition“ aus aktiven und ehemaligen Entscheidungsträgern (Fischer 2012), Intellektuellen (Habermas 2011) und eher links (Busch/Hirschel 2011) wie eher rechts stehenden Think-Tanks (Marzinotto/Sapir/Wolff 2011) fordert? Gerade die Politikwissenschaft kann hier Antworten liefern, die für die öffentliche Debatte

\section{Für diesen Hinweis danke ich Martin Höpner.}


erheblich relevanter sind als diejenigen der die öffentliche Meinungsbildung bisher dominierenden Wirtschaftswissenschaften. Dazu müsste sie sich aber auch unvoreingenommen und ohne Scheuklappen mit den hier nur sehr komprimiert skizzierten Themen beschäftigen.

Insgesamt kann als Fazit dieses vorläufigen Literaturberichts festgehalten werden, dass die von vielen Experten vorhergesagten Probleme eines nicht optimalen Währungsraums in der Tat eingetreten sind, wenn auch kaum jemand die tatsächliche Dynamik vorausgeahnt hat. Die Eurokrise hat in jedem Fall eine Vielzahl von Forschungsagenden aufgeworfen, die die Europaforschung noch lange beschäftigen werden und deren Beantwortung den systematischen Blick über disziplinäre Grenzen erfordert. Die fundamentale Frage wird dabei diejenige nach der problematischen Vereinbarkeit der europäischen Integration mit demokratischer Selbstbestimmung sein, und zwar vor allem im Kernbereich der politischen Ökonomie.

\section{Literatur}

Bearce, David, 2009: EMU. The Last Stand for the Policy Convergence Hypothesis?, in: Journal of European Public Policy 16 (4), 582-600.

Beckert, Jens/Streeck, Wolfgang, 2012: Die Fiskalkrise und die Einheit Europas, in: Aus Politik und Zeitgeschichte (4), 7-17.

Bieling, Hans-Jürgen, 2011: Vom Krisenmanagement zur neuen Konsolidierungsagenda der EU, in: Prokla 41 (2), 173-194.

Blyth, Mark/Abdelal, Rawi/Parsons, Craig, 2010: Constructing the International Economy, Ithaca (NY).

Blyth, Mark/Matthijs, Matthias, 2011: Why Only Germany Can Fix the Euro, in: Foreign Affairs, http://www.foreignaffairs.com/articles/136685/matthiasmatthijs-and-mark-blyth/why-only-germany-can-fix-the-euro 27.5.2012).

Bundeszentrale für politische Bildung (Hrsg.), 2012: Deutsche Außenpolitik, Aus Politik und Zeitgeschichte B 62 (10).

Busch, Klaus/Hirschel, Dierk, 2011: Europa am Scheideweg. Wege aus der Krise, Friedrich Ebert Stiftung, Bonn.

Busch, Andreas, 2010: Der Euro als Vorteil und als Nachteil für Deutschland, in: Aus Politik und Zeitgeschichte (43), 25-32.

Cafruny, Alan/Ryner, Magnus, 2009: Critical Political Economy, in: Antje Wiener/ Thomas Diez (Hrsg.), European Integration Theory, Oxford, 221-240.

Cooper, Ian, 2011: The euro crisis as the revenge of neo-functionalism, http://euobserver.com/7/113682 (Stand: 1.5.2012). 
De Grauwe, Paul, 2000: Economics of Monetary Union, New York (4. Aufl.).

Decker, Frank, 2011: Von der Euro-Krise zu neuen Ufern? Perspektiven des europäischen Integrationsprojekts, in: Zeitschrift für Politikwissenschaft 21 (2), 303-312.

Dyson, Kenneth, 2000: The Politics of the Euro-Zone, Oxford.

Dyson, Kenneth, 2008: The First Decade. Credibility, Identity and Institutional "Fuzziness", in: Kenneth Dyson (Hrsg.), The Euro at Ten, Oxford, 1-36.

Dyson, Kenneth/Featherstone, Kevin, 1999: The Road to Maastricht. Negotiating Economic and Monetary Union, Oxford.

Eichengreen, Barry, 2010: The Breakup of the Euro Area, in: Alberto Alesina/ Francesco Giavazzi (Hrsg.), Europe and the Euro, Chicago, 11-51.

Eichengreen, Barry, 2012: European Monetary Integration with the Benefit of Hindsight, in: Journal of Common Market Studies 50 (51), 123-136.

Enderlein, Henrik/Verdun, Amy, 2009: EMU's teenage challenge: what have we learned and can we predict from political science?, in: Journal of European Public Policy 16 (4), 490-507.

European Commission, 2008: EMU@10. Successes and challenges after ten years of Economic and Monetary Union, in: European Economy 2, Luxemburg.

Featherstone, Kevin, 2011: The Greek Sovereign Debt Crisis and EMU. A Failing State in a Skewed Regime, in: Journal of Common Market Studies 49 (2), 193-217.

Fischer, Joschka, 2012: Europa steht in Flammen, Süddeutsche Zeitung, 4.6.2012. Gottwald, Jörn Carsten, 2012: Regulating Financial Services in the EU: a case of successful institutional learning, in: Hubert Zimmermann/Andreas Dür (Hrsg.), Key Controversies in European Integration, Basingstoke, 131-137.

Graziano, Paolo/Vink, Maarten Peter (Hrsg.), 2006: Europeanization: New Research Agendas, New York.

Habermas, Jürgen, 2011: Zur Verfassung Europas. Ein Essay, Berlin.

Heipertz, Martin/Verdun, Amy, 2010: Ruling Europe: The Politics of the Stability and Growth Pact, Cambridge.

Heritier, Adrienne/Rhodes, Martin (Hrsg.), 2011: New Modes of Governance in Europe. Governing in the Shadow of Hierarchy, Basingstoke.

Hodson, Dermot, 2011: Governing the Euro Area in Good Times and Bad, Oxford. Höpner, Martin, 2009: Spielarten des Kapitalismus als Schule der vergleichenden Staatstätigkeitsforschung, in: Zeitschrift für Vergleichende Politikwissenschaft 3 (2), 407-415. 
Höpner, Martin/Schäfer Armin, 2010: Grenzen der Integration - wie die Intensivierung der Wirtschaftsintegration zur Gefahr für die politische Integration wird, in: Integration 33 (1), 3-20.

Höpner, Martin/Schäfer, Armin, 2012: Integration among Unequals. How the Heterogeneity of European Varieties of Capitalism Shapes the Social and Democratic Potential of the EU, in: Pablo Beramendi/Silja Häusermann/Herbert Kitschelt/Hanspeter Kriesi (Hrsg.), The Future of Democratic Capitalism (i. E.). Höpner, Martin/Schäfer, Armin/Zimmermann, Hubert, 2012: Erweiterung, Vertiefung und Demokratie. Das Trilemma der europäischen Integration, Frankfurter Allgemeine Zeitung, 27.4.2012,12.

Howarth, David/Loedel, Peter, 2005: The European Central Bank, Basingstoke (2. Aufl.).

Jones, Eric, 2010: Merkel's Folly, in: Survival 52 (3), 21-38.

Kaube, Jürgen, 2012: Weltmacht der Herzen. O je, die Europaforschung, in: Frankfurter Allgemeine Zeitung, 15.2.2012, N4.

Kohler-Koch, Beate/Eising, Rainer (Hrsg.), 1999: The Transformation of Governance in the European Union, London.

Lindberg, Leon N./Scheingold, Stuart A., 1970: Europe's Would-Be Polity: Patterns of Change in the European Community, Englewood Cliffs.

Lynn, Matthew, 2011: Bust. Greece, the Euro, and the Sovereign Debt Crisis, Hoboken.

Majone, Giandomenico, 2005: Dilemmas of European Integration. The Ambiguities and Pitfalls of Integration by Stealth, Oxford.

Marzinotto, Benedicta/Sapir, Andre/Wolff, Guntram, 2011: What Kind of Fiscal Union? Bruegel Policy Brief 06 (November).

McNamara, Kathleen, 1998: The Currency of Ideas, Ithaca (NY).

Moravcsik, Andrew, 1998: The Choice for Europe: Social Purpose and State Power from Messina to Maastricht, London.

Mügge, Daniel, 2012: The Pitfalls of EU Governance in Financial Markets, in: Hubert Zimmermann/Andreas Dür (Hrsg.), Key Controversies in European Integration, 138-144.

Nölke, Andreas, 2012: Rettet Europa! Ohne den Euro?, in: Blätter für deutsche und internationale Politik 3, 55-64.

Nölke, Andreas/Heires, Marcel, 2011: Finanzkrise und Finanzialisierung, in: Oliver Kessler (Hrsg.), Die Politische Ökonomie der Weltfinanzkrise, Wiesbaden, $37-52$. 
Pew Research Center, 2012: European Unity on the Rocks, http://www.pewglobal.org/files/2012/05/Pew-Global-Attitudes-Project-European-Crisis-Report-FINAL-FOR-PRINT-May-29-2012.pdf (Stand: 9.6.2012).

Puetter, Uwe, 2006: The Eurogroup. How a Secretive Circle of Finance Ministers Shapes European Economic Governance, Manchester.

Reinhart, Carmen M./Rogoff, Kenneth, 2009: This Time Is Different. Eight Centuries of Financial Folly, Princeton.

Rödl, Florian, 2012: EU im Notstandsmodus, in: Blätter für deutsche und internationale Politik 5, 5-8.

Sadeh, Tal/Verdun, Amy, 2009: Explaining Europe's Monetary Union - A Survey of the Literature, in: International Studies Review 11 (2), 277-301.

Salines, Marion/Glöckler, Gabriel/Truchlewski, Zbignew, 2012: Existential Crisis, Incremental Response: the Eurozone's Dual Institutional Evolution 2007-2011, in: Journal of European Public Policy 19 (5), 665-681.

Scharpf, Fritz, 2011: Monetary Union, Fiscal Crisis and the Pre-emption of Democracy, in: Zeitschrift für Staats- und Europawissenschaften 2, 163-198.

Scheller, Henrik, 2012: Fiscal Governance und Demokratie in Krisenzeiten, in: Aus Politik und Zeitgeschichte B 62, 9-16.

Schmitter, Phillip, 1997: The Emerging Euro-polity and its Impact upon National Systems of Production, in: Hollingsworth J.R. and Boyer Robert, Hrsg, Contemporary Capitalism: the Embeddedness of Institutions, Cambridge, 395-430.

Schwarzer, Daniela, 2012: Economic Governance in der Eurozone, in: Aus Politik und Zeitgeschichte B 62, 17-24.

Staatsprojekt Europa (Hrsg.), 2012: Die EU in der Krise. Zwischen autoritärem Etatismus und europäischem Frühling, Münster.

van Apeldoorn, Bastiaan/Drahokoupil, Jan/Horn, Laura (Hrsg.), 2009: Contradictions and Limits of Neoliberal European Governance. From Lisbon to Lisbon, Basingstoke.

Verdun, Amy, 2000: European Responses to Globalization and Financial Market Integration, Basingstoke.

Voßkuhle, Andreas, 2012: Über die Demokratie in Europa, in: Aus Politik und Zeitgeschichte B 62, 3-9.

Young, Brigitte/Semmler, Willi, 2011: The European Sovereign Debt Crisis. Is Germany to Blame?, in: German Politics \& Society 29 (1), 1-24.

Zimmermann, Hubert, 2009: Die EU und die Globale Finanzkrise 2008/2009, in: Politische Bildung 3, 63-80.

Zimmermann, Hubert, 2012 a: Constructing Contagion. Germany's Role in the Eurozone-Crisis, in: Perspectives on Europe 41 (2), 27-32. 
Zimmermann, Hubert, 2012 b: Die gnadenlose Euro-Logik, in: Blätter für deutsche und internationale Politik 1, 59-65.

Zimmermann, Hubert/Dür, Andreas (Hrsg.), 2012: Key Controversies in European Integration, Basingstoke.

Korrespondenzanschrift:

Prof. Dr. Hubert Zimmermann

Institut für Politikwissenschaft

Philipps Universität Marburg

Wilhelm-Röpke-Straße $6 \mathrm{G}$

35032 Marburg

E-Mail: hubert.zimmermann@staff.uni-marburg.de

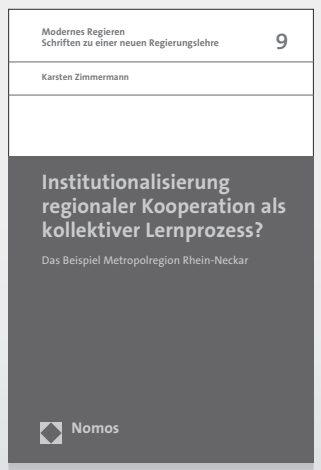

Institutionalisierung regionaler Kooperation als kollektiver Lernprozess?

Das Beispiel Metropolregion Rhein-Neckar

Von Karsten Zimmermann

2012, 335 S., brosch., 59,-€

ISBN 978-3-8329-6374-3

(Modernes Regieren - Schriften zu einer neuen

Regierungslehre, Bd. 9) 